\title{
Demographic, Anthropometric, and Biochemical Characteristics of Jordanian Women With Polycystic Ovary Syndrome: A Case-Control Study
}

\author{
Jehan Hamadneh ${ }^{1 *(1)}$, Nahla S. Al-bayyari², Shereen Hamadneh ${ }^{3}$, Safaa Al-Zeidaneen ${ }^{4}$, Hadil Subih'², \\ Samar Z. Burgan ${ }^{5}$, Zouhair O. Amarin ${ }^{1}$
}

\begin{abstract}
Objectives: To compare the demographic, anthropometric, and biochemical characteristics between women with polycystic ovary syndrome (PCOS) and healthy women and to identify the independent biochemical markers of PCOS.

Materials and Methods: A case-control study was conducted on 77 women with PCOS and 73 healthy controls aged between 18 and 49 years. Blood samples were obtained for the determination of plasma total homocysteine (tHcy), total cholesterol (TC), triglycerides (TG), low- and high-density lipoprotein, TC/high-density lipoprotein ratio, total testosterone (TT), sex hormone-binding globulin, and free androgen index (FAl). Finally, multivariable logistic regression analysis was performed to identify the independent biochemical markers of PCOS.

Results: The mean differences between cases and controls in $\mathrm{HCy}, \mathrm{TG}$, and sex hormone-binding globulin were statistically significant. tHcy and TG were elevated in cases while sex hormone-binding globulin demonstrated a reduction. All three markers were identified as independent predictors of PCOS in multivariable analysis in addition to a fourth, TT. tHcy is an independent biomarker of PCOS despite a relationship with TG and TC, the former of which is itself an independent biomarker.

Conclusions: In general, overweight women with PCOS had higher levels of tHcy and TG and a lower level of sex hormone-binding globulin compared with healthy controls. The level of tHcy is a marker of PCOS independent of the lipid profile. The differential biochemical profile of women with PCOS and healthy women provides useful hints for the diagnosis of PCOS and thus must be addressed in relation to the risk of cardiovascular disease.

Keywords: Polycystic ovary syndrome, Homocysteine, Androgens, Lipids
\end{abstract}

\section{Introduction}

Polycystic ovary syndrome (PCOS) is the most common disorder of the endocrine system, affecting $5 \%-10 \%$ of women of reproductive age worldwide $(1,2)$. Although the prevalence of this syndrome has increased significantly, its etiology is not completely understood yet $(2,3)$. PCOS is a multifactorial disorder in which genetic, hormonal, and environmental factors play significant roles (2).

This disorder is defined by signs and symptoms such as menstrual irregularity, hyperandrogenism, and polycystic ovarian morphology. The ultrasound definition of polycystic ovarian morphology is 12 or more follicles in each ovary measuring 2-9 $\mathrm{mm}$ in diameter (5). To establish the diagnosis of PCOS according to the Rotterdam criteria, at least two of the previously mentioned symptoms and signs must be present $(1,2)$. These criteria have been associated with problematic features such as infertility, miscarriages, preeclampsia, metabolic disorders (e.g., type 2 diabetes mellitus), and cardiovascular disease $(1,4)$.
Previous evidence shows that women with PCOS have a seven-fold increase in the risk of developing type 2 diabetes mellitus. In other words, $33 \%$ of women with PCOS develop glucose intolerance and about $10 \%$ of them develop type 2 diabetes mellitus early in life (4). According to some studies (6-8), these women are also more prone to other metabolic aberrations including hyperhomocysteinemia, hyperandrogenism, and elevated triglycerides (TG), total cholesterol (TC), and low-density lipoprotein (LDL). In addition, women with PCOS, particularly those with elevated homocysteine levels, may have reduced high-density lipoprotein (HDL) and sex hormone-binding globulin (SHBG) levels (2). It has been advocated that women of reproductive age should undergo regular testing to prevent these complications (4).

Nonetheless, the demographic, anthropometric, and biochemical characteristics of Jordanian women with PCOS have not so far received attention. Therefore, this study had to aims including comparing demographic, 
Key Messages

- Overweight women with polycystic ovary syndrome (PCOS) had higher levels of plasma total homocysteine (tHcy) and triglycerides (TG), as well as, lower level of sex hormone-binding globulin compared with healthy women.

- Biochemical profile of women with PCOS provides useful hints for the diagnosis of PCOS and thus must be addressed in relation to the risk of cardiovascular disease and developing type 2 diabetes mellitus.

- Adjusting lifestyle and nutritional factors is an important factor to improve reproductive health, healthcare providers should consider this factor, especially for women with PCOS.

anthropometric, and biochemical characteristics between women with PCOS and healthy women of reproductive age and then identifying the independent biochemical markers of PCOS.

\section{Materials and Methods}

This case-control study was conducted on 150 women (77 cases of PCOS and 73 healthy controls), aged between 18 and 49 years, who attended the obstetrics and gynecology clinics at the King Abdullah University Hospital (KAUH) in the North of Jordan from December 2016 to February 2017.

\section{Participants}

The included cases were overweight women with a body mass index (BMI) of $25-29.99 \mathrm{~kg} / \mathrm{m}^{2}$, aged between 18 49 years old, diagnosed with PCOS according to the Rotterdam criteria, non-smokers, and not diagnosed with any chronic diseases. On the other hand, women were excluded from the study if they were aged $<18$ years or $>49$ years, pregnant, lactating, underweight, of normal weight, obese, smokers, diagnosed with a chronic illness, on lipidlowering medications, or vitamins supplementations. Controls were healthy women who attended the obstetrics and gynecology clinics for a routine checkup.

\section{Procedure}

Each participant was interviewed using a standardized data collection form to obtain information on demographics, current medication, use of vitamin supplements, smoking, and alcohol consumption. Body weight and height were measured, and BMI was calculated as well. After an overnight fast of at least 12 hours, a venous blood sample was obtained from the forearm of each participant by a trained researcher. Blood samples were left at room temperature for 30 minutes before centrifugation. Then, the obtained sera from blood samples were labeled, stored in Eppendorf tubes at $-70^{\circ} \mathrm{C}$, and used for determining TC, TG, LDL, HDL, TC/HDL ratio, total testosterone (TT), SHBG, and the free androgen index (FAI). Plasma total homocysteine (tHcy) was measured by liquid chromatography-tandem mass spectrophotometry. Each sample was tested for homocysteine twice and the average of the two readings was taken as well. According to the results of previous studies, tHcy $\leq 10 \mu \mathrm{mol} / \mathrm{L}$ was considered normal and levels $>10 \mu \mathrm{mol} / \mathrm{L}$ were interpreted as hyperhomocysteinemia. Serum TC, LDL, HDL, TG, SHBG, TT, and free testosterone levels were measured in the diagnostic laboratory of KAUH. TC $\geq 5.18 \mathrm{mmol} / \mathrm{L}$, $\mathrm{LDL} \geq 3.37 \mathrm{mmol} / \mathrm{L}, \mathrm{HDL}<1.04 \mathrm{mmol} / \mathrm{L}$, and TG $\geq 1.7$ $\mathrm{mmol} / \mathrm{L}$ were considered as hypercholesterolemia, a high level, low, and hypertriglyceridemia, respectively. Eventually, FAI was calculated by dividing the level of TT on the SHBG level multiplied by 100 .

\section{Data Analysis}

Data were entered using IBM SPSS Statistics (version 22). All variables were tested for normality, and descriptive statistics were generated accordingly. Frequencies and percentages were used to describe categorical variables while means and standard errors were applied to describe continuous variables. Participants were stratified according to the tHcy level $(\leq 10 \mu \mathrm{mol} / \mathrm{L}$ or $>10 \mu \mathrm{mol} / \mathrm{L})$. The difference between the two groups regarding the normally distributed means of anthropometric and biochemical variables was compared using the independent-samples $t$ test while the $\chi^{2}$ test and Fisher exact test, as appropriate, were used to test the associations of the groups with the demographic variables. Multivariable logistic regression analysis was employed to identify the biochemical predictors of the case-control status, and a value of $P<0.05$ was considered statistically significant.

\section{Results}

A total of 150 study participants were included in the final analysis. The mean age of the participants was $28.87 \pm$ 0.74 years. The mean weight, height, and BMI were 70.29 \pm 0.81 kilograms, $159.87 \pm 0.65$ centimeters, and 27.39 $\pm 0.15 \mathrm{~kg} / \mathrm{m}^{2}$, respectively. Eighty-five study participants (56.7\%) were married, 92 (61.3\%) of them were highly educated, and $114(76 \%)$ cases had a monthly family income of no more than 1000 Jordanian dinars (equivalent to approximately 1,400 United States dollars). The mean values of the biochemical characteristics were $18.84 \pm 1.09$ $\mu \mathrm{mol} / \mathrm{L}, 1.31 \pm 0.07 \mathrm{mmol} / \mathrm{L}, 1.26 \pm 0.02 \mathrm{mmol} / \mathrm{L}, 3.44 \pm$ $0.07 \mathrm{mmol} / \mathrm{L}, 5.20 \pm 0.08 \mathrm{mmol} / \mathrm{L}, 4.28 \pm 0.10,1.54 \pm 0.06$ $\mathrm{nmol} / \mathrm{L}, 79.30 \pm 5.70 \mathrm{nmol} / \mathrm{L}$, and $4.03 \pm 0.46$ for total tHcy, TG, HDL, LDL, TC, the TC:HDL ratio, TT, SHBG, and the FAI, respectively.

The case-control status was statistically significantly associated with marital status and occupation, but not the city of residence, level of education, or monthly family income (Table 1). There was a statistically significant mean difference between cases and controls in terms of age and the levels of tHcy, TG, and SHBG (Table 2). Compared to the controls, study cases were younger, their tHcy and TG levels were elevated, and their SHBG 
Table 1. Demographic Characteristics of the Study Participants Comprising Women With Polycystic Ovary Syndrome (Cases) and Healthy Women (Controls)

\begin{tabular}{|c|c|c|c|c|}
\hline \multirow{2}{*}{ Variable } & \multicolumn{3}{|c|}{ Group } & \multirow{2}{*}{$P$ Value } \\
\hline & Total $(\mathrm{N}=150)$ & Cases $(n=77)$ & Controls $(n=73)$ & \\
\hline \multicolumn{4}{|l|}{ Marital status } & \multirow{3}{*}{$0.001^{*}$} \\
\hline Married & $85(56.7)$ & $28(36.4)$ & $57(78.1)$ & \\
\hline Single & $65(43.3)$ & $49(63.6)$ & $16(21.9)$ & \\
\hline \multicolumn{4}{|l|}{ City of residence } & \multirow{3}{*}{0.327} \\
\hline Irbid & $120(80.0)$ & $60(77.9)$ & $60(82.2)$ & \\
\hline Other & $30(20.0)$ & $17(22.1)$ & $13(17.8)$ & \\
\hline \multicolumn{4}{|l|}{ Occupation } & \multirow{3}{*}{$0.001^{*}$} \\
\hline Student & $50(33.3)$ & $38(49.4)$ & $12(16.4)$ & \\
\hline Other & $100(66.7)$ & $39(50.6)$ & $61(83.6)$ & \\
\hline \multicolumn{4}{|l|}{ Level of education } & \multirow{3}{*}{0.076} \\
\hline Educated & $58(38.7)$ & $25(32.5)$ & $33(45.2)$ & \\
\hline Highly educated & $92(61.3)$ & $52(67.5)$ & $40(54.8)$ & \\
\hline \multicolumn{4}{|l|}{ Monthly family income (JD) } & \multirow{3}{*}{0.503} \\
\hline$<1000$ & $114(76.0)$ & $59(76.6)$ & $55(75.3)$ & \\
\hline$>1000$ & $36(24.0)$ & $18(23.4)$ & $18(24.7)$ & \\
\hline
\end{tabular}

Note. Values are presented as absolute $(\mathrm{n})$ and relative $(\%)$ frequencies. $* P \leq 0.05$ is statistically significant.

level represented a reduction. The levels of TG and TC also differed between all study participants according to their tHcy level $(\leq 10 \mu \mathrm{mol} / \mathrm{L}$ or $>10 \mu \mathrm{mol} / \mathrm{L})$ while the remaining biomarkers demonstrated no difference in this regard (Table 3). Both TG and TC levels increased in study participants with a tHcy level $>0 \mu \mathrm{mol} / \mathrm{L}$. The same pattern was observed in a case-only analysis of tHcy. In other words, the levels of TG and TC indicated statistically significant differences according to the $\mathrm{tHcy}$ level $(\leq 10 \mu \mathrm{mol} / \mathrm{L}$ or $>10 \mu \mathrm{mol} / \mathrm{L})$, but the remaining biomarkers failed to show such a difference (Table 4).

Multivariable logistic regression revealed four significant biochemical predictors of case-control status, namely, the

Table 2. Mean Comparisons of Age, Anthropometric, and Biochemical Characteristics Between Polycystic Ovary Syndrome Cases and Healthy Controls

\begin{tabular}{lccc}
\hline \multirow{2}{*}{ Variable } & \multicolumn{2}{c}{ Group } & \multirow{2}{*}{ P Value } \\
\cline { 2 - 3 } & Cases $(\mathbf{n}=\mathbf{2 6})$ & Controls $(\mathbf{n}=\mathbf{1 2 4})$ & \\
\hline Age $(\mathrm{y})$ & $23.58 \pm 0.55$ & $34.45 \pm 1.07$ & $0.001^{*}$ \\
Weight $(\mathrm{kg})$ & $68.81 \pm 1.20$ & $71.85 \pm 1.06$ & 0.060 \\
Height $(\mathrm{cm})$ & $158.80 \pm 0.93$ & $161.01 \pm 0.88$ & 0.085 \\
$\mathrm{BMI}\left(\mathrm{kg} / \mathrm{m}^{2}\right)$ & $27.12 \pm 0.20$ & $27.67 \pm 0.22$ & 0.065 \\
tHcy $(\mu \mathrm{mol} / \mathrm{L})$ & $21.3 \pm 1.88$ & $16.26 \pm 0.97$ & $0.021^{*}$ \\
TG $(\mathrm{mmol} / \mathrm{L})$ & $1.47 \pm 0.12$ & $1.14 \pm 0.07$ & $0.015^{*}$ \\
$\mathrm{HDL}(\mathrm{mmol} / \mathrm{L})$ & $1.26 \pm 0.03$ & $1.27 \pm 0.03$ & 0.822 \\
$\mathrm{LDL}(\mathrm{mmol} / \mathrm{L})$ & $3.53 \pm 0.10$ & $3.36 \pm 0.10$ & 0.229 \\
$\mathrm{TC}(\mathrm{mmol} / \mathrm{L})$ & $5.26 \pm 0.11$ & $5.14 \pm 0.11$ & 0.442 \\
$\mathrm{TC} / \mathrm{HDL} \mathrm{ratio}$ & $4.40 \pm 0.14$ & $4.16 \pm 0.15$ & 0.237 \\
$\mathrm{TT}(\mathrm{nmol} / \mathrm{L})$ & $1.64 \pm 0.09$ & $1.43 \pm 0.08$ & 0.088 \\
$\mathrm{SHBG}(\mathrm{nmol} / \mathrm{L})$ & $61.30 \pm 4.98$ & $96.37 \pm 9.70$ & $0.002^{*}$ \\
FAI & $4.06 \pm 0.58$ & $4.01 \pm 0.73$ & 0.956 \\
\hline
\end{tabular}

Note. BMI: Body mass index; tHcy: Plasma total homocysteine; TG: Triglycerides; HDL: high-density lipoprotein; LDL: low-density lipoprotein; TC: Total cholesterol; TT: Total testosterone; SHBG: Sex hormone-binding globulin; FAl: Free androgen index. Values are presented as means and standard errors. ${ }^{*} P \leq 0.05$ is statistically significant. levels of tHcy, TG, TT, and SHBG (Table 5). Women with a tHcy level $>10 \mu \mathrm{mol} / \mathrm{L}$ were 3.11 times more likely to be cases compared with women with a tHcy level $\leq 10 \mu \mathrm{mol} / \mathrm{L}$ $(P=0.032)$. Women with a TG level $>1.7 \mathrm{mmol} / \mathrm{L}$ were 2.74 times more likely to be cases in comparison with women with a TG level $<1.7 \mathrm{mmol} / \mathrm{L}(P=0.009)$. The likelihood of a woman with a TT level $>3.1 \mathrm{nmol} / \mathrm{L}$ belonging to the case group was exactly two-fold higher than a woman with a TT level $<3.1 \mathrm{nmol} / \mathrm{L}$. Finally, the likelihood of a woman with an SHBG level $\leq 135.5 \mathrm{nmol} / \mathrm{L}$ belonging to the case group was 1.01-fold higher when compared to a woman with an SHBG level $>135.5 \mathrm{nmol} / \mathrm{L}(P=0.004)$.

Table 3. Mean Comparisons of Age Anthropometric and Biochemical Characteristics of the Study Participants According to Homocysteine Concentration

\begin{tabular}{lccc}
\hline \multirow{2}{*}{ Variable } & \multicolumn{2}{c}{ tHcy $(\boldsymbol{\mu m o l} / \mathbf{L})$} & \multirow{2}{*}{$\boldsymbol{P}$ Value } \\
\cline { 2 - 3 } & \multicolumn{1}{c}{$\mathbf{1 0}(\mathbf{n}=\mathbf{1 1})$} & $\mathbf{1 0}(\mathbf{n}=\mathbf{6 6})$ & \\
\hline Age $(\mathrm{y})$ & $31.58 \pm 2.06$ & $28.25 \pm 0.77$ & 0.065 \\
Weight $(\mathrm{kg})$ & $70.90 \pm 2.05$ & $70.16 \pm 0.88$ & 0.729 \\
Height $(\mathrm{cm})$ & $160.81 \pm 1.70$ & $159.68 \pm 0.70$ & 0.509 \\
BMI $\left(\mathrm{kg} / \mathrm{m}^{2}\right)$ & $27.28 \pm 0.32$ & $27.41 \pm 0.17$ & 0.748 \\
TG $(\mathrm{mmol} / \mathrm{L})$ & $1.22 \pm 0.06$ & $1.71 \pm 0.28$ & $0.007^{*}$ \\
HDL $(\mathrm{mmol} / \mathrm{L})$ & $1.28 \pm 0.06$ & $1.26 \pm 0.02$ & 0.688 \\
LDL $(\mathrm{mmol} / \mathrm{L})$ & $3.40 \pm 0.07$ & $3.70 \pm 0.19$ & 0.088 \\
TC $(\mathrm{mmol} / \mathrm{L})$ & $5.12 \pm 0.08$ & $5.60 \pm 0.23$ & $0.023^{*}$ \\
TC/HDL ratio & $4.24 \pm 0.10$ & $4.48 \pm 0.35$ & 0.347 \\
TT $(\mathrm{nmol} / \mathrm{L})$ & $1.52 \pm 0.06$ & $1.61 \pm 0.19$ & 0.546 \\
SHBG $(\mathrm{nmol} / \mathrm{L})$ & $94.88 \pm 17.01$ & $76.04 \pm 5.90$ & 0.212 \\
FAI & $3.98 \pm 0.50$ & $4.30 \pm 1.30$ & 0.794 \\
\hline
\end{tabular}

Total number of participants $=150$.

Note. BMI: Body mass index; tHcy: Plasma total homocysteine; TG: Triglycerides; HDL: high-density lipoprotein; LDL: low-density lipoprotein; TC: Total cholesterol; TT: Total testosterone; SHBG: Sex hormone-binding globulin; FAI: Free androgen index. Values are presented as means and standard errors. ${ }^{*} P \leq 0.05$ is statistically significant. 
Table 4. Mean Comparisons of Age Anthropometric and Biochemical Characteristics of Women With Polycystic Ovary Syndrome According to Plasma Total Homocysteine Concentration

\begin{tabular}{|c|c|c|c|}
\hline \multirow{2}{*}{ Variable } & \multicolumn{2}{|c|}{ tHcy $(\mu \mathrm{mol} / \mathrm{L})$} & \multirow{2}{*}{$P$ Value } \\
\hline & $<10(n=11)$ & $>10(n=66)$ & \\
\hline Age $(y)$ & $22.55 \pm 1.11$ & $23.76 \pm 0.62$ & 0.445 \\
\hline Weight (kg) & $68.68 \pm 3.27$ & $68.83 \pm 1.30$ & 0.967 \\
\hline Height $(\mathrm{cm})$ & $158.82 \pm 2.50$ & $158.79 \pm 1.00$ & 0.991 \\
\hline $\mathrm{BMI}\left(\mathrm{kg} / \mathrm{m}^{2}\right)$ & $27.08 \pm 0.41$ & $27.12 \pm 0.22$ & 0.941 \\
\hline $\mathrm{TG}(\mathrm{mmol} / \mathrm{L})$ & $1.35 \pm 0.09$ & $2.17 \pm 0.60$ & $0.012^{*}$ \\
\hline $\mathrm{HDL}(\mathrm{mmol} / \mathrm{L})$ & $1.32 \pm 0.08$ & $1.25 \pm 0.03$ & 0.459 \\
\hline LDL (mmol/L) & $3.28 \pm 0.09$ & $3.83 \pm 0.30$ & $0.043^{*}$ \\
\hline $\mathrm{TC}(\mathrm{mmol} / \mathrm{L})$ & $5.14 \pm 0.11$ & $6.03 \pm 0.35$ & $0.006^{*}$ \\
\hline TC/HDL ratio & $4.32 \pm 0.13$ & $4.85 \pm 0.54$ & 0.173 \\
\hline $\mathrm{TT}(\mathrm{nmol} / \mathrm{L})$ & $1.31 \pm 0.32$ & $1.69 \pm 0.09$ & 0.140 \\
\hline $\mathrm{SHBG}(\mathrm{nmol} / \mathrm{L})$ & $131.75 \pm 33.15$ & $90.47 \pm 9.82$ & 0.137 \\
\hline FAI & $3.94 \pm 0.50$ & $4.76 \pm 2.95$ & 0.629 \\
\hline
\end{tabular}

Note. BMI: Body mass index; tHcy: Plasma total homocysteine; TG: Triglycerides; HDL: high-density lipoprotein; LDL: low-density lipoprotein; TC: Total cholesterol; TT: Total testosterone; SHBG: Sex hormone-binding globulin; FAI: Free androgen index. Values are presented as means and standard errors. ${ }^{*} P \leq 0.05$ is statistically significant.

Table 5. Multivariable Logistic Regression Model for the Biochemical Predictors of Case-control Status (Polycystic Ovary Syndrome or Healthy)

\begin{tabular}{lcccc}
\hline Variable & b & SE & P Value & OR $(\mathbf{9 5} \% \mathbf{C l})$ \\
\hline tHcy $>10 \mu \mathrm{mol} / \mathrm{L}$ & 1.133 & 0.528 & $0.032^{*}$ & $3.106(1.103-8.744)$ \\
$\mathrm{TG}>1.7 \mathrm{mmol} / \mathrm{L}$ & 1.007 & 0.384 & $0.009^{*}$ & $2.736(1.289-5.809)$ \\
$\mathrm{TC}>5.2 \mathrm{mmol} / \mathrm{L}$ & -0.077 & 0.221 & 0.728 & $0.926(0.601-1.428)$ \\
$\mathrm{BMI}>27.99$ & -0.204 & 0.123 & 0.096 & $0.815(0.641-1.037)$ \\
$\mathrm{TT}>3.1 \mathrm{nmol} / \mathrm{L}$ & 0.693 & 0.296 & $0.019^{*}$ & $2.000(1.120-3.574)$ \\
$\mathrm{SHBG} \leq 135.5 \mathrm{nmol} / \mathrm{L}$ & 0.010 & 0.004 & $0.004^{*}$ & $1.010(1.003-1.017)$ \\
$\mathrm{FAl}>5.89$ & 0.025 & 0.039 & 0.514 & $1.026(0.950-1.107)$ \\
Constant & 1.912 & 3.315 & 0.564 & 6.767 \\
\hline NOte. & & & $\mathrm{TC}$ &
\end{tabular}

Note. tHcy: Plasma total homocysteine; TG: Triglycerides; TC: Total cholesterol; BMI: Body mass index; TT: Total testosterone; SHBG: Sex hormone-binding globulin; FAI: Free androgen index; Hosmer and Lemeshow test: $\chi^{2}=13.332 ; \mathrm{df}=8 ; P$ value $=0.101 ; \mathrm{b}:$ Regression coefficient; SE: Standard error of the regression coefficient; OR: Odds ratio; $95 \% \mathrm{Cl}: 95 \%$ confidence interval; ${ }^{*} P \leq 0.05$ is statistically significant.

\section{Discussion}

In our study, the mean tHcy level was higher in women with PCOS compared to healthy women, and the difference was statistically significant. Our findings are similar to the results of previous studies (9-15). In addition, the mean comparison of SHBG levels was statistically significant. However, the mean level was lower in women with PCOS in comparison with healthy women, which is in line with the results of other studies $(16,17)$. However, the findings of a previous study demonstrated a relationship between PCOS and the other markers of hyperandrogenism, namely, the TT level and the FAI (17), which contradicts the findings of the current study. Although in this study, the TT level was an independent biochemical marker in multivariable analysis, the lack of a statistically significant bivariable relationship implies that the reading may be masked by interactions with other biomarkers in the clinic. In another study, the results revealed no meaningful difference in the SHBG level between women with PCOS and healthy controls. In addition, no significant difference was found in the TG level between the groups (18). In contrast, the results of the present study represented that the TG level was a differential marker, which is in conformity with the findings of a previous study (19).

The level of tHcy in our study participants was directly related to the levels of TG and TC, but none of the markers of hyperandrogenism. The same results were observed in a subgroup analysis of women with PCOS instead of the entire sample. These relationships have previously been investigated in the context of other conditions. For example, the findings of a community-based study showed that the level of tHcy is inversely related to HDL-C and directly related to TG (20). The latter relationship was observed in the present study. In another study on patients with myocardial infarction, it was found that the tHcy level is negatively correlated with HDL-C while positively correlated with LDL-C (21). The relationship between tHcy and lipids was also demonstrated in further studies $(22,23)$. Based on the results of one study, BMI and waistto-hip ratio may be used as surrogate markers of tHcy (24). Despite this relationship, which may explain the association of tHcy with PCOS, our multivariable analysis revealed that tHcy is a differential biomarker between cases and controls independent of the lipid profile. Finally, previous studies have consistently reported that the tHcy level in women with PCOS is not related to the degree of obesity, insulin resistance, or androgen levels $(4,25)$, which conforms to the findings of the current study. Furthermore, behavioral fertility disorders are common in Jordan. Thus, it is strongly recommended for adjusting lifestyle and nutritional factors to improve reproductive health (26).

The main limitation of the study relates to the generalizability of the results. Namely, our sampling method was nonrandom and thus it was impossible to make population-level inferences. However, our sample was drawn from an understudied ethnic group. In addition, this study did not assess dietary markers, including folic acid and vitamin B12. Future studies should include dietary markers, a wider biochemical panel, and a larger sample size in order to detect the relationships of the weak-to-moderate strength. Future studies in this ethnic group should also evaluate the cardiovascular risk associated with the metabolic dysfunction of PCOS.

In conclusion, women with PCOS have higher levels of tHcy and TG while a lower level of SHBG compared with healthy controls. The differential biochemical profile of women with PCOS and healthy women provides useful hints for the diagnosis of PCOS and should be addressed in relation to cardiovascular disease. 


\section{Authors' Contribution}

$\mathrm{JH}$ and NSA conceived and designed the study. $\mathrm{JH}, \mathrm{NSA}$, and $\mathrm{SH}$ reviewed the literature and collected the data. $\mathrm{JH}$ and NSA conducted data analysis and interpreted the results. $\mathrm{SH}$ provided technical support. JH, NSA, SH, SA, HS, ZOA, and SZB drafted the manuscript. JH supervised the entire study process.

\section{Conflict of Interests}

Authors declare that they have no conflict of interests.

\section{Ethical Issues}

All procedures performed in this study involving human participants were in accordance with the ethical standards of the Institutional Review Board at Jordan University of Science and Technology and the King Abdullah University Hospital.

\section{Financial Support}

The study was supported by a grant from the Jordan University of Science and Technology.

\section{References}

1. Goodman NF, Cobin RH, Futterweit W, Glueck JS, Legro RS, Carmina E. American Association of Endocrinologists, American College of Endocrinology, and Androgen Excess and PCOS Scoiety Disease State Clinical Review: Guide to The Best Practices in The Evaluation and Treatment of Polycystic Ovary Syndrome - Part 1. Endocr Pract. 2015;21(11):1291-1300. doi:10.4158/ep15748.dsc

2. De Leo V, Musacchio MC, Cappelli V, Massaro MG, Morgante G, Petraglia F. Genetic, hormonal and metabolic aspects of PCOS: an update. Reprod Biol Endocrinol. 2016;14(1):38. doi:10.1186/ s12958-016-0173-x

3. El-Gharib MN. Hyperhomocysteinemia and polycystic ovary syndrome. Reprod Med Int. 2018;1(1):1-3. doi:10.23937/rmi2017/1710003

4. Hart R, Doherty DA. The potential implications of a PCOS diagnosis on a woman's long-term health using data linkage. J Clin Endocrinol Metab. 2015;100(3):911-919. doi:10.1210/jc.2014-3886

5. Hasan JS, Ali AH, Fakhrildin MB. Assessment of homocysteine level in plasma of Iraqi women with polycystic ovary syndrome and insulin resistance. Global Journal of Bio-Science and Biotechnology. 2015;4(2):191-194.

6. Thomson RL, Spedding S, Buckley JD. Vitamin D in the aetiology and management of polycystic ovary syndrome. Clin Endocrinol (Oxf). 2012;77(3):343-350. doi:10.1111/j.1365-2265.2012.04434.x

7. Ardabili HR, Gargari BP, Farzadi L. Vitamin D supplementation has no effect on insulin resistance assessment in women with polycystic ovary syndrome and vitamin D deficiency. Nutr Res. 2012;32(3):195-201. doi:10.1016/j.nutres.2012.02.001

8. Hassan NE, El-Orabi HA, Eid YM, Mohammed NR. Effect of 25-hydroxyvitamin $\mathrm{D}$ on metabolic parameters and insulin resistance in patients with polycystic ovarian syndrome. Middle East Fertil Soc J. 2012;17(3):176-180. doi:10.1016/j.mefs.2012.04.005

9. Loverro G, Lorusso F, Mei L, Depalo R, Cormio G, Selvaggi L. The plasma homocysteine levels are increased in polycystic ovary syndrome. Gynecol Obstet Invest. 2002;53(3):157-162. doi:10.1159/000058367

10. Badawy A, State O, El Gawad SSA, El Aziz OA. Plasma homocysteine and polycystic ovary syndrome: the missed link. Eur J Obstet Gynecol Reprod Biol. 2007;131(1):68-72. doi:10.1016/j.ejogrb.2006.10.015

11. Maleedhu P, Vijayabhaskar M, Sharma SSB, Kodumuri PK, Devi VD. Status of Homocysteine in Polycystic Ovary Syndrome (PCOS). J Clin Diagn Res. 2014;8(2):31-33. doi:10.7860/jcdr/2014/7070.3999

12. Devi SL, Abdul Jaweed S. Homocysteine, C-reactive protein and traditional cardiovascular risk markers in polycystic ovary syndrome.
Int J Cur Res Rev. 2013;5(6):59-68.

13. Orio F Jr, Palomba S, Di Biase S, et al. Homocysteine levels and C677T polymorphism of methylenetetrahydrofolate reductase in women with polycystic ovary syndrome. J Clin Endocrinol Metab. 2003;88(2):673-679. doi:10.1210/jc.2002-021142

14. Boulman N, Levy $\mathrm{Y}$, Leiba $\mathrm{R}$, et al. Increased C-reactive protein levels in the polycystic ovary syndrome: a marker of cardiovascular disease. J Clin Endocrinol Metab. 2004;89(5):2160-2165. doi:10.1210/jc.2003-031096

15. Schachter M, Raziel A, Friedler S, Strassburger D, Bern O, Ron-El R. Insulin resistance in patients with polycystic ovary syndrome is associated with elevated plasma homocysteine. Hum Reprod. 2003;18(4):721-727. doi:10.1093/humrep/deg190

16. Mancini F, Cianciosi A, Reggiani GM, Facchinetti F, Battaglia C, de Aloysio D. Endothelial function and its relationship to leptin, homocysteine, and insulin resistance in lean and overweight eumenorrheic women and PCOS patients: a pilot study. Fertil Steril. 2009;91(6):2537-2544. doi:10.1016/j.fertnstert.2008.03.023

17. Yuan C, Liu X, Mao Y, Diao F, Cui Y, Liu J. Polycystic ovary syndrome patients with high BMI tend to have functional disorders of androgen excess: a prospective study. J Biomed Res. 2016;30(3):197-202. doi:10.7555/jbr.30.20140111

18. Esmaeilzadeh S, Tahmasbpour E, Gholinezhad-Chari M. Hyperhomocysteinemia, insulin resistance and body mass index in Iranian young women with polycystic ovary syndrome. Middle East Fertil Soc J. 2017;22(2):149-155. doi:10.1016/j.mefs.2017.01.007

19. Panda SR, Rout PK, Chandra C. A case control study of role of lipid profile in polycystic ovarian syndrome: is there any role in non-obese polycystic ovary syndrome? Int J Reprod Contracept Obstet Gynecol. 2016;5(6):1981-1984. doi:10.18203/2320-1770. ijrcog20161702.

20. Momin M, Jia J, Fan F, et al. Relationship between plasma homocysteine level and lipid profiles in a community-based Chinese population. Lipids Health Dis. 2017;16(1):54. doi:10.1186/ s12944-017-0441-6

21. Qujeq D, Omran TS, Hosini L. Correlation between total homocysteine, low-density lipoprotein cholesterol and high-density lipoprotein cholesterol in the serum of patients with myocardial infarction. Clin Biochem. 2001;34(2):97-101. doi:10.1016/s00099120(01)00187-4

22. Yadav AS, Bhagwat VR, Rathod IM. Relationship of plasma homocysteine with lipid profile parameters in ischemic heart disease. Indian J Clin Biochem. 2006;21(1):106-110. doi:10.1007/ bf02913076

23. de Luis DA, Fernandez N, Arranz ML, Aller R, Izaola O, Romero E. Total homocysteine levels relation with chronic complications of diabetes, body composition, and other cardiovascular risk factors in a population of patients with diabetes mellitus type 2. J Diabetes Complications. 2005;19(1):42-46. doi:10.1016/j. jdiacomp.2003.12.003

24. Al-Bayyari N, Hamadneh J, Hailat R, Hamadneh S. Total homocysteine is positively correlated with body mass index, waistto-hip ratio, and fat mass among overweight reproductive women: a cross-sectional study. Nutr Res. 2017;48:9-15. doi:10.1016/j. nutres.2017.10.008

25. Meng Y, Chen X, Peng Z, Liu X, Sun Y, Dai S. Association between high serum homocysteine levels and biochemical characteristics in women with polycystic ovarian syndrome: a systematic review and meta-analysis. PLoS One. 2016;11(6):e0157389. doi:10.1371/ journal.pone.0157389

26. Hamadneh JM, Amarin Z, Hamadneh S, Obeidat NA, Kassab M, Bani Hani M. Lifestyle related fertility disorders in north Jordan: potential for improvement. Int J Womens Health Reprod Sci. 2017;5(4):264-269. doi:10.15296/ijwhr.2017.45

(C) 2021 The Author(s); This is an open-access article distributed under the terms of the Creative Commons Attribution License (http:// creativecommons.org/licenses/by/4.0), which permits unrestricted use, distribution, and reproduction in any medium, provided the original work is properly cited. 